\title{
Vasopressin, Angiotensin II and Tyrosine-Hydroxylase Expression in the Hypothalamus of the Syndrome of Inappropriate ADH: A Case Report
}

\author{
Emilia M. Carmona-Calero, ${ }^{1,2}$, Leandro Castañeyra-Ruiz ${ }^{2}$, Ibrahim Gonzalez-Marrero ${ }^{1}$, \\ Juan M. Gonzalez-Toledo ${ }^{1}$, Hector De Paz-Carmona ${ }^{2}$, Agustín Castañeyra-Ruiz ${ }^{2}$, \\ Maria L. Ruiz-Mayor ${ }^{1}$ and Agustin Castañeyra-Perdomo ${ }^{*}, 1,2$ \\ ${ }^{I}$ Departamento de Anatomía, Anatomía Patológica e Histología, Facultad de Medicina, Universidad de La Laguna, La \\ Laguna, Tenerife, Canary Island, Spain \\ ${ }^{2}$ Instituto de Investigación y Ciencias de Puerto del Rosario, Puerto del Rosario, Fuerteventura, Canary Island, Spain
}

\begin{abstract}
The Syndrome of Inappropriate Antidiuretic Hormone (SIADH) is a disease characterized by hyponatremia and hyperosmolality of urine, and where vasopressin, angiotensin II and catecholamines are implicated in salt water balance, cardiovascular and blood pressure regulation. Therefore, the aim of this study is to analyze the hypothalamic distribution of vasopressin (VAS) Angiotensin II (AGII) and tyrosine-hydroxylase (TH) in a case of SIADH and compare it with a case without SIADH. Two hypothalamus taken from a 66 year-old man with SIADH, and the other from a 63 year-old man without SIADH, were used. An immunohistochemical study was performed using anti-VAS, anti-AGII and anti-TH as primary antibodies. The vasopressin immunoreactive material was mainly found in the supraoptic nucleus (SON) and paraventricular nucleus (PVN), but VAS was also shown in the periventricular nucleus, preoptic medial area, perifornical nucleus and hypothalamic lateral area. The AGII was also mainly found in the SON and PVN. The TH was found in the SON, PVN, arcuate nucleus, periventricular nucleus, perifornical nucleus, preoptic medial and lateral area. VAS was less intensive and in a lesser number of cells and fibres in the case of SIADH, as opposed to the AGII and TH which were similar in both cases.
\end{abstract}

Keywords: SIADH, vasopressin, angiotensin II, tyrosine-hydroxylase.

\section{INTRODUCTION}

The first case of a patient with the Syndrome of Inappropriate Antidiuretic Hormone (SIADH) was reported by Schwartz et al. [1] who described two patients with lung cancer who developed hyponatremia associated with continuous sodium loss in urine. They postulated that the tumors led to the release of inappropriate antidiuretic hormone (ADH). The clinical description of the syndrome has changed little since the original article, therefore the Syndrome of Inappropriate Antidiuretic Hormone Secretion (SIADH) is a disease characterized by hyponatremia and hyperosmolality of urine [2,3]. There are well known causes of this syndrome, such as neoplasmatic processes, different disorders of the central nervous system, lung diseases and the side effects of various drugs. Therefore, studies [4] of larger series of patients have revealed that SIADH occurs in $3 \%$ of patients with head and neck cancer, in $0.7 \%$ of patients with non-small-cell lung cancer, and in $15 \%$ of cases of small-cell lung cancer [4]. The optimal therapy for SIADH is to treat the underlying malignant disease. If this is not possible, or if the disease has become refractory, other treatment methods are available such as water restriction, demeclocycline therapy, or, in severe cases, infusion of

*Address correspondence to this author at the Departamento de Anatomía, Anatomía Patológica e Histología, Facultad de Medicina, Universidad de La Laguna, 38071 La Laguna, Tenerife, Canary Island, Spain;

Tel: 34 922319352; Fax: 34922660253; E-mail: acastane@ull.es hypertonic saline together with furosemide during treatment [4]. Total body water and tonicity is strictly regulated by the renal action of the antidiuretic hormone (ADH), reni angiotensin-aldosterone system, norepinephrine and by the thirst mechanism. Abnormalities in water balance are manifested in SIADH as sodium disturbances-hyponatremia and hypernatremia [4]. On the other hand, the presence of VAS, AGII and TH and their implication in cardiovascular, salt water balance and blood pressure regulation have long been described in the hypothalamus in man and different animal species [5-8]. Several author have also described the detection of tyrosine hydroxylase-immunoreactivity and vasopressin mRNA and ANGII in the hypothalamus that could be related with the hypertension and SIADH [9-12]. The aim of the present work is to analyze the hypothalamic distribution of vasopressin (VAS), AGII and Tyrosinehydroxylase (TH) in a case of SIADH.

\section{CASE REPORT}

Two hypothalamus taken from two human brains from the collection of the Anatomy Department of the University of La Laguna were studied here. A 66 year old man that had developed hyponatremia and showed all the symptoms of the Syndrome of Inappropriate Antidiuretic Hormone Secretion (SIADH), and the other subject was a 63 year old man without SIADH as a control. Non-small-cell lung cancer was the cause of death in the SIADH case, in the other case the underlying disease was cardioangioesclerosis and the patient died of multi-organ failure. The hypothalamus were cut in 
four series of coronal sections $10 \mu \mathrm{m}$ thick. The A series was stained with the Klüver-Barrier method. The B, C and D series were processed immunohistochemically; antivasopressin (VAS) (ICN Biomedicals, Inc. Catal.6\#:4717), anti-angiotensin II (AGII) [7] and anti-TyrosineHydroxylase (TH)(SIGMA, T 2928) were used as primary antibodies. The anti-VAS, the anti-AGII and the anti-TH were diluted at 1:2000, 1:100 and 1:1000 respectively in PBS-Triton with $2 \%$ normal goat serum, the incubation was for $24 \mathrm{~h}$, at room temperature. Followed by "DAKO StreptABCcomplex/HRP Duet, Mouse/Rabbit" procedure. The reaction product was visualized by diaminobenzidine reaction. Method specificity was controlled by omitting the primary antibody.
The immunohistochemistry slides were converted to digital images by using an LEICA DMRB photomicroscope with an LEICA DC $300 \mathrm{~F}$ camera (Gemany). Image analysis was completed in Image $\mathrm{J}(\mathrm{v} .1 .43 \mathrm{u}, \mathrm{NIH}$, Bethesda, MD, USA). The 'Mean Gray Value' was measured from the selected nuclei for all stained tissue. This value gives the average stain intensity in grayscale units for all threshold pixels. A single-factor analysis of variance (ANOVA) was used for the immunohistochemistry statistical study, which was conducted using the IBM SPSS statistic 19 software.

The vasopressin immunoreactive material (VAS-ir) was mainly found in both cases (Figs. 1, 2) in the rostral, intermediate and caudal part of the supraoptic hypothalamic nucleus and magnocellular part of the entering
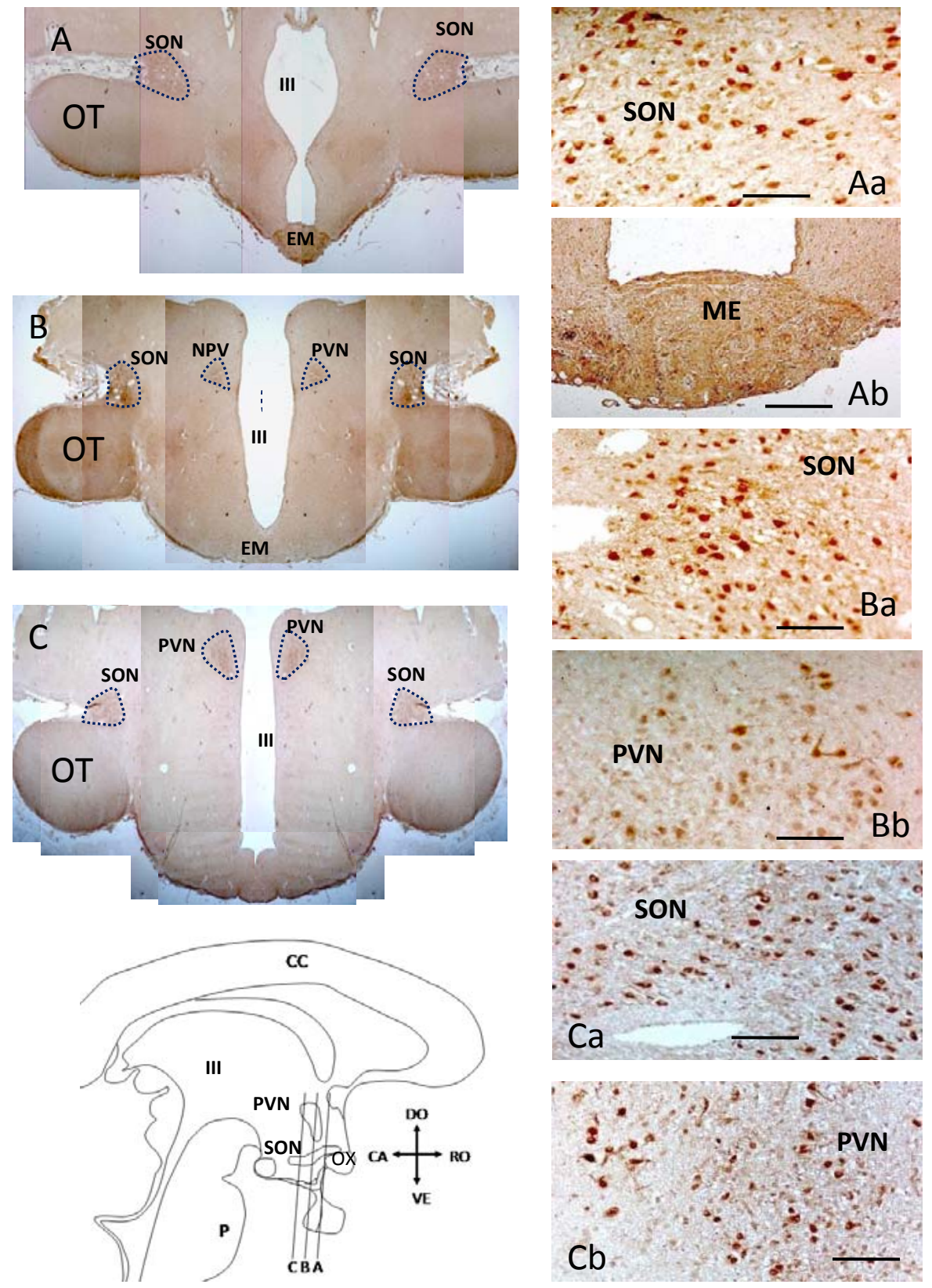

Fig. (1). Rostro-caudal frontal section photographs composition $(\mathbf{A}, \mathbf{B}, \mathbf{C})$ of the human hypothalamus with SIADH, immunostained with anti-vasopressin. $\mathrm{ME}=$ median eminence, $\mathrm{PVN}=$ paraventricular nucleus, $\mathrm{SON}=$ supraoptic nucleus, $\mathrm{III}=3^{\mathrm{er}}$ ventricle, $\mathrm{OX}=$ optic chiasm, $\mathrm{CC}=$ corpus callosum, $\mathrm{OT}=$ optic tract, $\mathrm{P}=$ Pons, $\mathrm{CA}=$ caudal, $\mathrm{RO}=$ rostral, $\mathrm{DO}=$ dorsal, $\mathrm{VE}=$ ventral $; \mathrm{Bar}=\mathrm{Aa}, \mathrm{Ba}, \mathrm{Bb}, \mathrm{Ca}, \mathrm{Cb} 100 \mu \mathrm{m}$, $\mathrm{Ab}=150 \mu \mathrm{m}$, 


\section{VAS}

NO SIADH
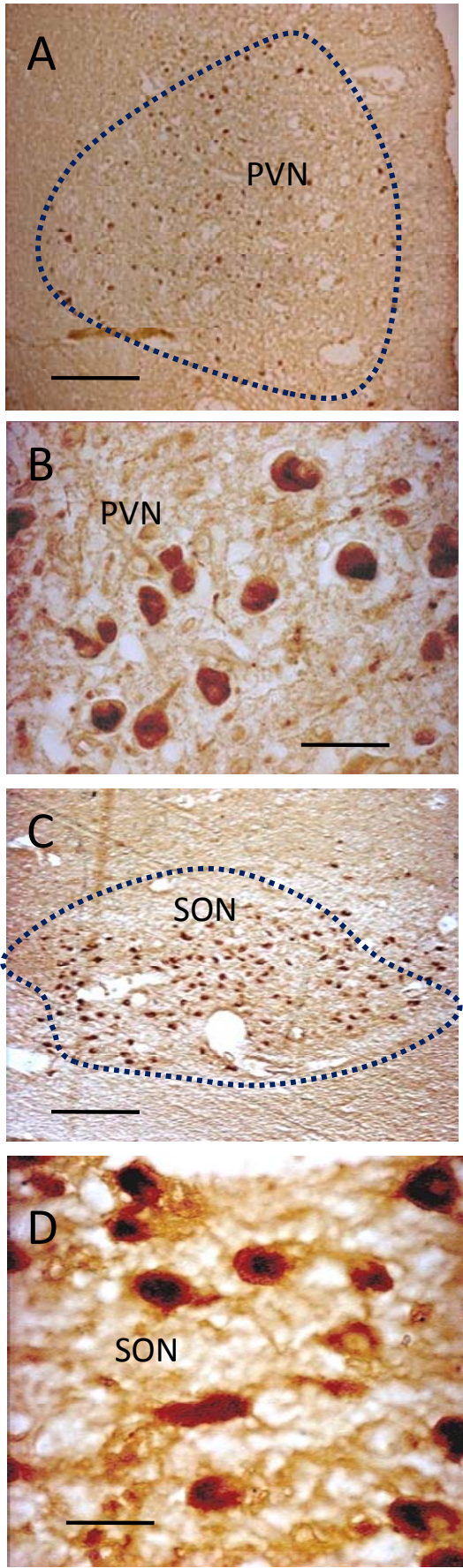

SIADH
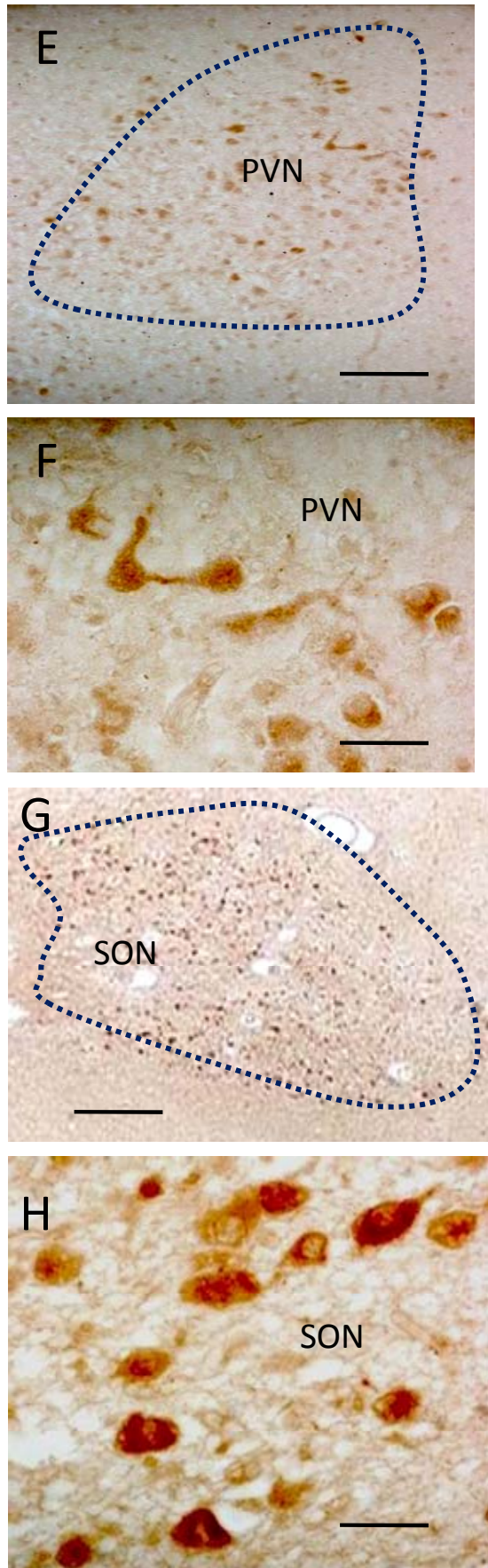

Fig. (2). Frontal section photographs of the human hypothalamus immunostained with anti-vasopressin. A, B, C, D without SIADH; E, F, G, H with SIADH. PVN: paraventricular nucleus, SON: supraoptic nucleus, III: 3er ventricle, VAS: vasopressin; Bar $=\mathbf{A}, \mathbf{G} 200 \mu \mathrm{m}, \mathbf{C}, \mathbf{E} 150$ $\mu \mathrm{m}, \mathbf{B}, \mathbf{F} 60 \mu \mathrm{m}, \mathbf{D}, \mathbf{H} 40 \mu \mathrm{m}$.

paraventricular hypothalamic nucleus, but VAS-ir was also shown in the periventricular nucleus, preoptic medial area, perifornical nucleus, and the caudal part of the hypothalamic lateral area. The AGII-ir was found (Fig. 3) in similar locations as VAS, but mainly in neurons of the SON and magnocellular part of the PVN. The tyrosine-hydroxylase immunoreactive (TH-ir) was found in SON and in PVN (Fig. 4), and also in the arcuate nucleus, periventricular nucleus, perifornical nucleus, preoptic medial and lateral area. TH-ir was higher in the PVN with respect to the SON.

VAS-ir (Graphs 1 and 2) was clearly decreased and in a smaller number of cells and fibres in SIADH, as opposed to the intensity of AGII-ir and TH-ir which were similar in both cases (Graphs 1 and 2) but TH-ir was clearly greater in both cases in the PVN with respect to the SON (Graph 3). 
NO SIADH
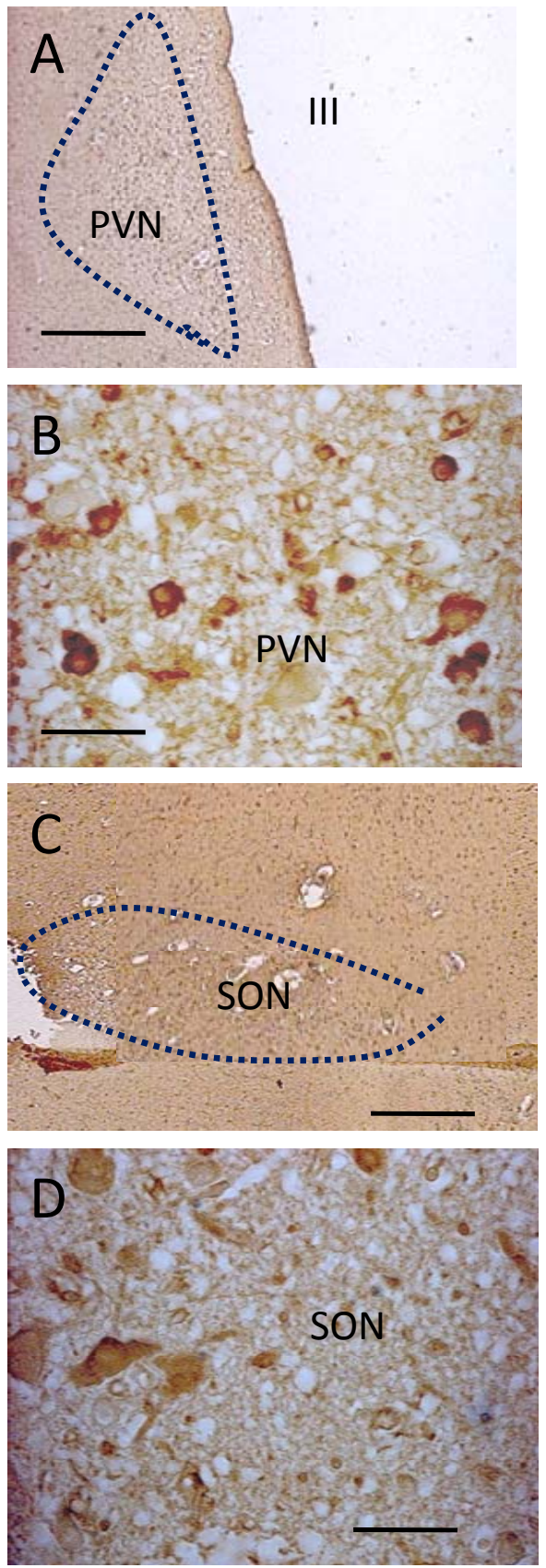

\section{SIADH}
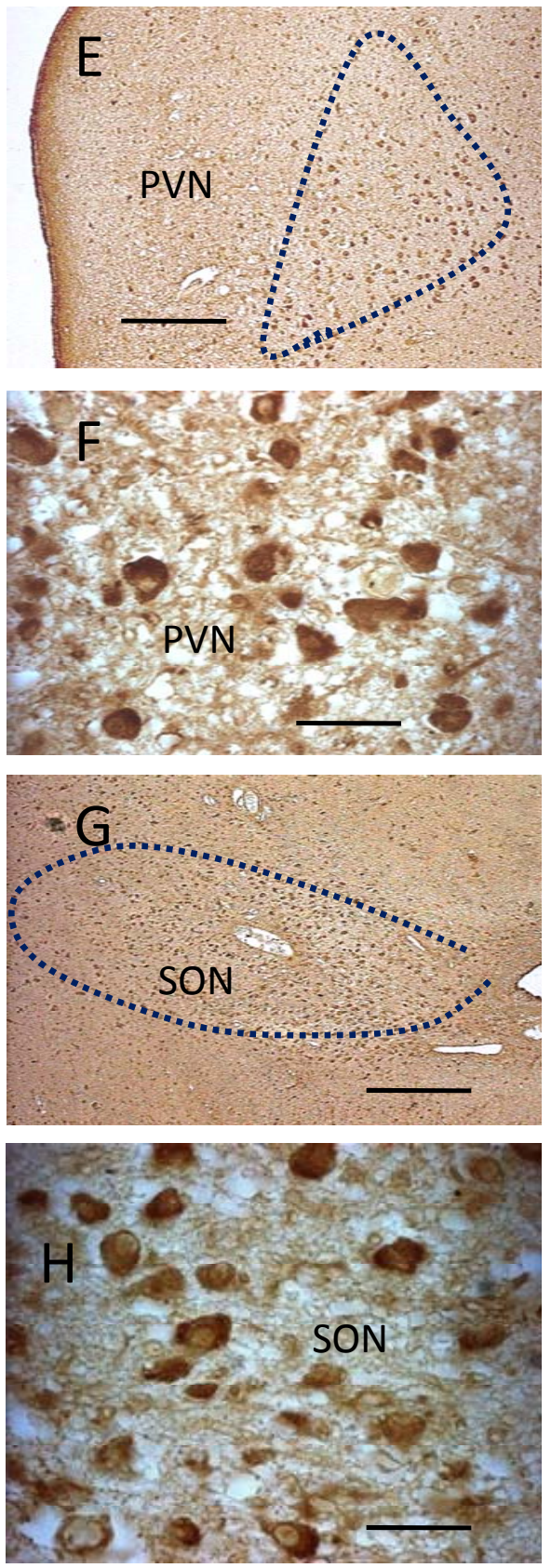

Fig. (3). Frontal section photographs of the human hypothalamus immunostained with anti-angiotensin II (AGII). A, B, C, D without SIADH; E, F, G, H with SIADH. PVN: paraventricular nucleus, SON: supraoptic nucleus, III: 3er ventricle, AGII: angiotensin II; Bar = A, C, G $250 \mu \mathrm{m}, \mathbf{E} 200 \mu \mathrm{m}, \mathbf{B}$, F $60 \mu \mathrm{m}, \mathbf{D}, \mathbf{H} 40 \mu \mathrm{m}$.

\section{DISCUSSION}

The Syndrome of Inappropriate Antidiuretic Hormone Secretion or Syndrome of Inappropriate Antidiuresis, is apparently induced by an extensive range of diseases, drugs, and/or injuries and is divisible into 3 kinds of abnormal vasopressin (VAS) release during hypertonic saline infusion [13]: type A, elevated, inconsistent fluctuations unrelated to increases in plasma sodium; type $\mathrm{B}$, a slow regular release, that is also unaltered by increases in plasma sodium; type C, a quick progressive increase in plasma VAS that closely correlates with plasma sodium as it rises towards the normal range; there is also another kind, type $\mathrm{D}$, that appears in a low percentage of patients, where there is no demonstrable defect in the osmoregulation of AVP and the cause of inappropriate antidiuresis is imprecise $[13,14]$. 


\section{$\mathrm{TH}$}

\section{NO SIADH}
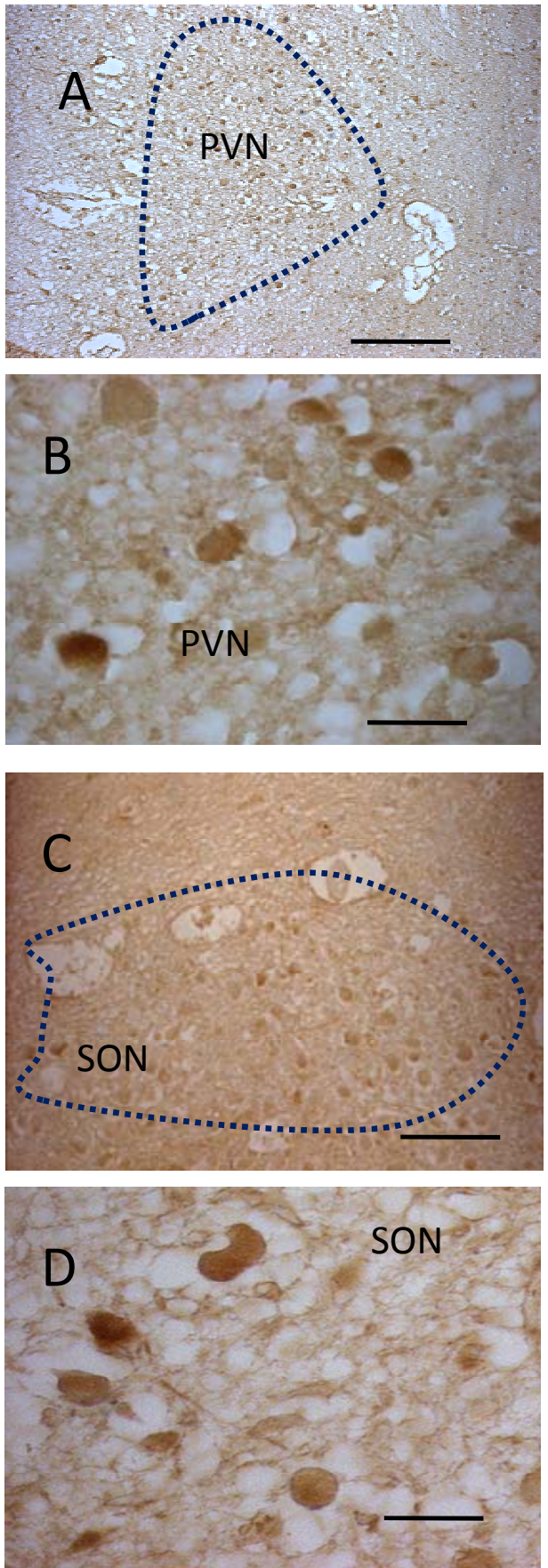

\section{SIADH}
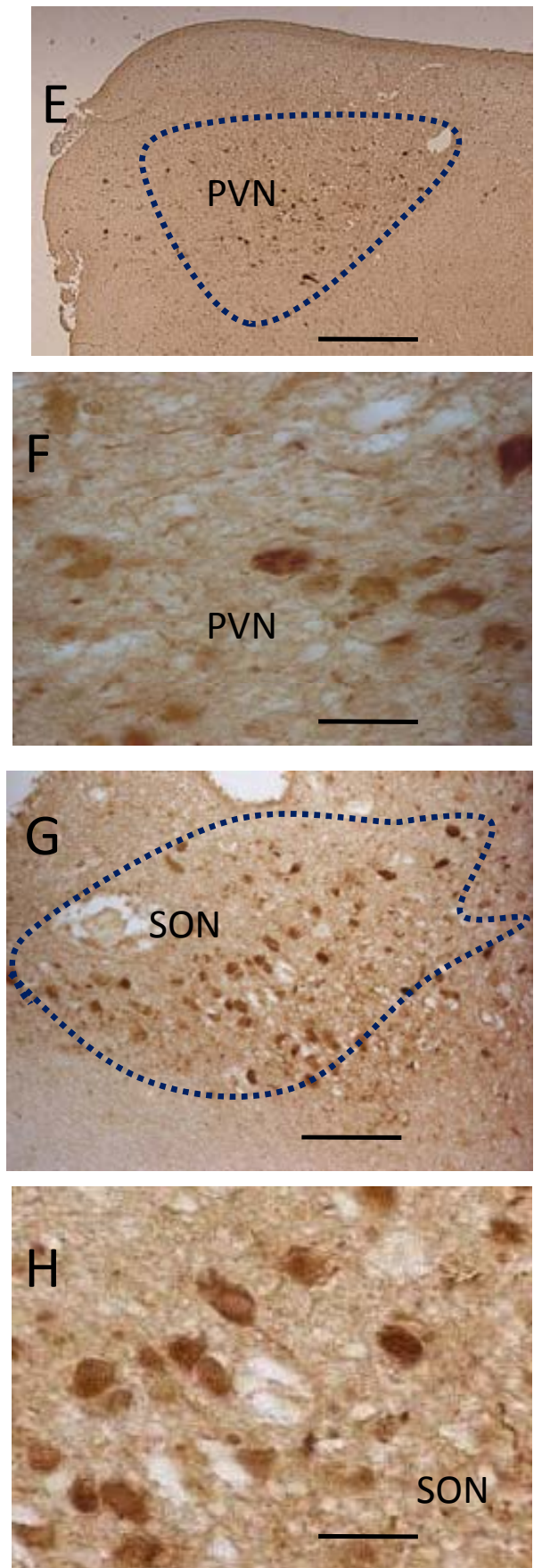

Fig. (4). Frontal section photographs of the human hypothalamus immunostained with anti- tyrosine-hydroxylase. A, B, C, D without SIADH; E, F, G, H with SIADH. PVN: paraventricular nucleus, SON: supraoptic nucleus, III: 3er ventricle, TH: tyrosine-hydroxylase; Bar $=\mathbf{A}, \mathbf{C}, \mathbf{G} 250 \mu \mathrm{m}, \mathbf{E} 200 \mu \mathrm{m}, \mathbf{B}, \mathbf{F} 60 \mu \mathrm{m}, \mathbf{D}, \mathbf{H} 40 \mu \mathrm{m}$.

The case presented in this paper could correspond to SIADH type C described above [13,14], because the patient periodically showed a progressive increase in plasma vasopressin that closely correlated with increased plasma sodium, and at other times the patient showed normal or decreased levels of VAS that corresponded to normal or decreased levels of plasma sodium.
On the other hand, several authors [14] have reported that the pathophysiological basis of SIADH is an absolute increase in body water. This increase depends on an excessive water intake that overwhelms the restricted renal ability of diluting urine and mounting compensatory dieresis due to vasopressin dysregulation, in agreement with this, the vasopressin immunoreactive material, in the present study, 


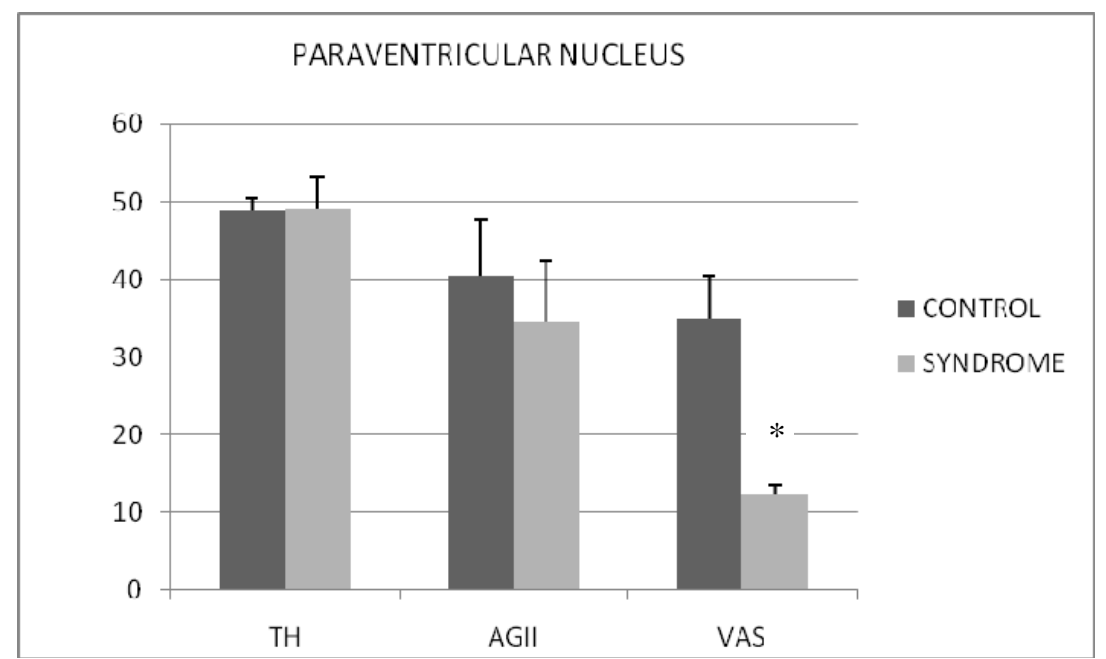

$* \mathrm{~F}_{1-6}=46,83 \quad \mathrm{p}<0,05$

Graph 1. The densitometry of vasopressin (VAS), angiotensin II (AGII) and tyrosine-hydroxylase (TH) immunoreactive in the hypothalamic paraventricular nucleous, * significant differences $F_{1-6}=46,83 \mathrm{p}<0,05$. Syndrome: case with SIADH, control: case without SIADH.

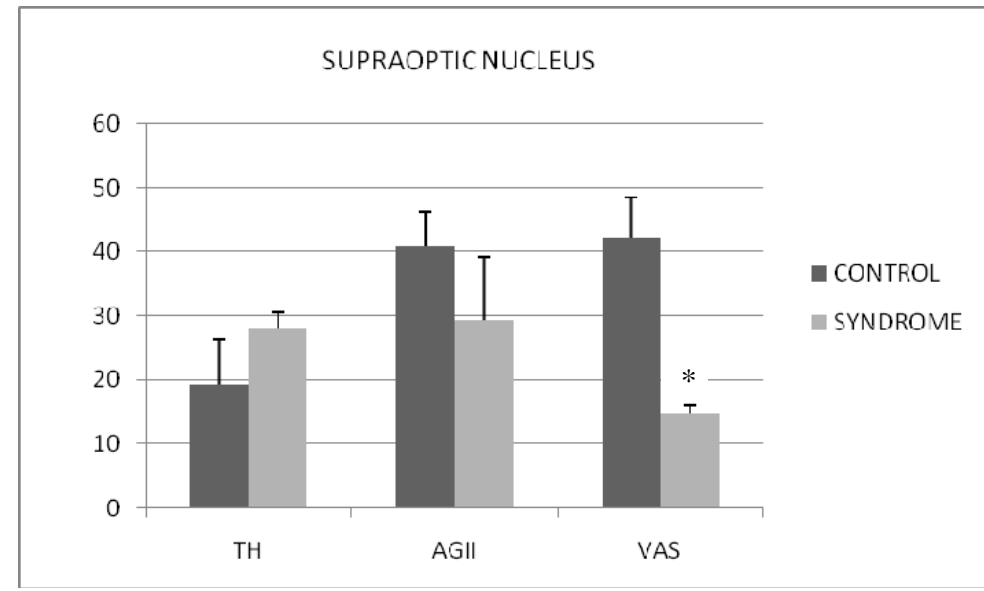

$* \mathrm{~F}_{1-6}=57,07 \quad \mathrm{p}<0,05$

Graph 2. The densitometry of vasopressin (VAS), angiotensin II (AGII) and tyrosine-hydroxylase (TH) immunoreactive in the hypothalamic supraoptic nucleous, * significant differences $\mathrm{F}_{1-6}=57,07 \mathrm{p}<0,05$. Syndrome: case with SIADH, control: case without SIADH

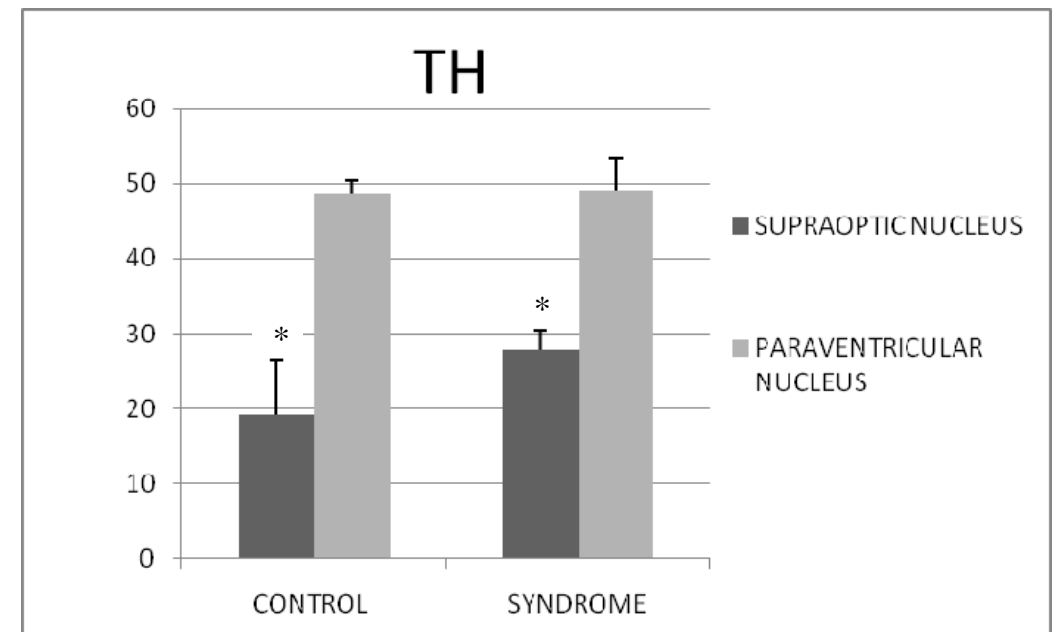

Control: case without $\operatorname{SIADH}{ }^{*} \mathrm{~F}_{1-6}=47,53 \mathrm{p}<0,05$; Syndrome: case of $\operatorname{SIADH}{ }^{*} \mathrm{~F}_{1-6}=55,00 \mathrm{p}<0,05$

Graph 3. The densitometry of vasopressin immunoreactive (VAS) in supraoptic (SON) and paraventricular (PVN) nucleus, * significant differences, control case without SIADH: $F_{1-6}=47,53 p<0,05$ Syndrome case with SIADH: $F_{1-6}=55,00 p<0,05$. 
was less intensive and in a lesser number of cells and fibres in the SIADH and was mainly found in the whole supraoptic nucleus and magnocellular part of the paraventricular hypothalamic nucleus. Vasopressin was also observed in the other hypothalamic areas. The decrease of VAS in the case of SIADH could mean an increase of the VAS release owing to variations in the vasopressin receptor, since other studies [15] have described some cases, whose clinical presentation and laboratory findings were consistent with SIADH, but which exhibited unmeasurable arginine-vasopressin (AVP) levels on repeated occasions that appears to be due to an activating mutation of the vasopressin receptor V2 (V2R). But, in other patients, this may be due to abnormal control of aquaporin-2 water channels in renal collecting tubules or the production of an antidiuretic principle other than VAS [13].

In conclusion, no differences in the AGII-ir and TH-ir intensity and location expression between both cases were found in the results here and only a decrease of VAS in both the paraventricular and supraoptic nucleus was observed, which could mean a lesser participation of TH and AGII than VAS in the physiopathology of the SIADH. These disparate kinds of results underlying SIADH may result in clear differences in its clinical presentation as well as its response to different kinds of treatment.

\section{ACKNOWLEDGEMENTS}

This work was supported by the Fundación Canaria de Instituto de Investigacion y Ciencias de Puerto del Rosario (INIPRO) projects: $01 / 10$ and $n^{\circ} 02 / 10$.

\section{CONFLICT OF INTEREST}

Declared none.

\section{REFERENCES}

[1] Schwartz WB, Bennett W, Curelop S, Bartter FC. A syndrome of renal sodium loss and hyponatremia probably resulting from inappropriate secretion of antidiuretic hormone. Am J Med 1957; 23: 529-42.

[2] Cabaj-Wiater I, Jakubowska I. Hyponatremia in syndrome of inappropiate antidiuretic hormone secretion--cases report. Pol Merkur Lekarski 2008; 25: 247-49.
[3] Decaux G, Musch W. Clinical laboratory evaluation of the syndrome of inappropriate secretion of antidiuretic hormone. Clin J Am Soc Nephrol 2008; 3: 1175-84.

[4] Sørensen JB, Andersen MK, Hansen HH. Syndrome of inappropriate secretion of antidiuretic hormone (SIADH) in malignant disease. J Intern Med 1995; 238: 97-110.

[5] de Wardener HE. The hypothalamus and hypertension. Physiol Rev 2001; 81: 1599-658.

[6] Ferrari MF, Coelho EF, Farizatto KL, Chadi G, Fior-Chadi DR. Modulation of tyrosine hydroxylase, neuropeptide y, glutamate, and substance $p$ in Ganglia and brain areas involved in cardiovascular control after chronic exposure to nicotine. Int $\mathrm{J}$ Hypertens 2011; 2011: 216464.

[7] Carmona-Calero EM, Pérez-González H, Martínez-Peña y Valenzuela I, et al. Effect of the arterial hypertension and captopril treatment on the angiotensin II content in the subfornical organ. A study in SHR rats. Histol Histopathol 2005; 20: 135-8.

[8] González-Marrero I, Castañeyra-Ruiz L, De Paz-Carmona $H$ Castañeyra-Ruiz A, González-Toledo JM, Carmona-Calero EM. Variaciones del sistema angiotensina-vasopresina hipotalámico ante hipertensión arterial y su tratamiento con captopril. Majorensis 2008; 4: 15-8.

[9] Mayinger B, Hensen J. Nonpeptide vasopressin antagonists: a new group of hormone blockers entering the scene. Exp Clin Endocrinol Diabetes 1999; 107(3): 157-65.

[10] Panayotacopoulou MT, Goudsmit E, Van Heerikhuize JJ, Swaab DF. Simultaneous detection of tyrosine hydroxylase-immunoreactivity and vasopressin mRNA in neurons of the human paraventricular and supraoptic nucleus. Brain Res 2000; 855(1): 181-5.

[11] Macova M, Pavel J, Saavedra JM. A peripherally administered, centrally acting angiotensin II AT2 antagonist selectively increases brain AT1 receptors and decreases brain tyrosine hydroxylase transcription, pituitary vasopressin and ACTH. Brain Res 2009; 1250: 130-40.

[12] Kaneko K, Shioya T, Yabuta K. Inappropriate secretion of antidiuretic hormone and transient hypertension associated with Guillain-Barré syndrome. Pediatr Neurosci 1989; 15(5): 257-9.

[13] Robertson GL. Regulation of arginine vasopressin in the syndrome of inappropriate antidiuresis. Am J Med 2006; 119 (7 Suppl 1): S36-42.

[14] Esposito P, Piotti G, Bianzina S, Malul Y, Dal Canton A. The syndrome of inappropriate antidiuresis: pathophysiology, clinical management and new therapeutic options. Nephron Clin Pract 2011; 119(1): c62-73.

[15] Gitelman SE, Feldman BJ, Rosenthal SM. Nephrogenic syndrome of inappropriate antidiuresisa novel disorder in water balance in pediatric patients. Am J Med 2006; 119 (Suppl 7A): S54-S8. 